\title{
Object-based selection: The role of attentional shifts
}

\author{
DOMINIQUE LAMY \\ Tel Aviv University, Ramat Aviv, Israel \\ and \\ HOWARD EGETH \\ Johns Hopkins University, Baltimore, Maryland
}

\begin{abstract}
The objective of this paper was to investigate under what conditions object-based effects are observed. Recently, Watson and Kramer (1999) used a divided-attention task and showed that unless topdown factors induce a bias toward selection at a higher level, object-based effects are obtained when same-object targets belong to the same uniformly connected (single-UC) region, but not when they belong to different single-UC regions grouped into a higher order object (grouped-UC regions). We refine this claim by proposing that a critical factor in determining whether or not object-based effects with grouped-UC regions are observed is the need to shift attention. The results of four experiments support this hypothesis. Stimuli and displays were similar to those used by Egly, Driver, and Rafal (1994). Subjects had to make size judgments. Using different paradigms, we obtained object-based effects when the task required shifts of attention (spatial cuing, same vs. different judgment with asynchronous target onsets), but not when attention remained either broadly distributed (same vs. different judgment with simultaneous targets) or tightly focused (response competition paradigm).
\end{abstract}

Over the last decade, much research has been devoted to the debate between space-based and object-based accounts of attentional selection (for reviews, see Egeth \& Yantis, 1997; Kanwisher \& Driver, 1992; Lamy \& Tsal, 2001). According to the space-based view, covert visual attention is directed to regions of space (e.g., Broadbent, 1982; Downing \& Pinker, 1985; Eriksen \& Hoffman, 1973; LaBerge \& Brown, 1989; Posner, Snyder, \& Davidson, 1980), whereas according to the object-based view, attention is directed to perceptual groups derived from the segmentation of the visual field according to Gestalt principles (e.g., Bundesen, 1990; Duncan, 1984; Duncan \& Humphreys, 1989, 1992; Kahneman \& Treisman, 1984; Neisser, 1967; Treisman, Kahneman, \& Burkell, 1983).

Evidence in support of the idea that attention selects perceptual groups, rather than unparsed regions of space, has been obtained using various paradigms. With dividedattention tasks, judging two attributes is easier when these belong to the same rather than to different perceptual groups (e.g., Baylis \& Driver, 1993; Behrmann, Zemel, \& Mozer, 1998, but see Saiki, 2000; Duncan, 1984; Lavie \& Driver, 1996, Experiments 1-3; Treisman et al., 1983; Vecera \& Farah, 1994; Watson \& Kramer, 1999). With Eriksen's flanker or response competition task (Eriksen \&

Support for this article was provided by NIMH Grant MH57388 to H.E. We thank Andy Leber for useful comments. Correspondence concerning this article should be addressed to D. Lamy, Department of Psychology, Tel Aviv University, Ramat-Aviv, POB 39040, Tel Aviv 69978, Israel (e-mail: domi@freud.tau.ac.il).
Hoffman, 1973), distractors grouped with the target are more difficult to ignore than distractors that belong to a different perceptual group (e.g., Baylis \& Driver, 1992; Driver \& Baylis, 1989; Kramer \& Jacobson, 1991). In spatial-cuing tasks, subjects are faster to redirect their attention within the same object than to another object (e.g., Abrams \& Law, 2000; Egly, Driver, \& Rafal, 1994; Lamy \& Tsal, 2000; Moore, Yantis, \& Vaughan, 1998; Vecera, 1994).

There seems to be little controversy that selection can be object based, and the fact that object-based effects have been obtained with a large array of different tasks and stimuli strongly suggests that the phenomenon is robust. However, little systematic manipulation of task and stimulus factors has been conducted to investigate whether there may be boundary conditions to object-based selection. Typically, each research group has arrived at its own specific combination of task, stimuli, and grouping principles that yields object-based effects. There have been three notable exceptions to this trend.

\section{Object-Based Selection and Task Requirements}

Vecera and Farah (1994) proposed that whether selection is space based or object based depends on task requirements. Specifically, they suggested that selection is object based only when the task requires shape judgments, whereas space-based selection prevails when object shape is irrelevant (e.g., in detection tasks). They used Duncan's (1984) stimuli: a box and a line superimposed on it. In the first two experiments, they found an object-based cost, replicating 
Duncan's finding that subjects are poorer at reporting two attributes when these belong to different objects, rather than to the same object, even when these objects occupy the same region in space. Vecera and Farah also manipulated the distance between the two objects, which were either superimposed, as in Duncan's original experiment, or distant from each other. They found the object-based cost to be independent of the distance between the two objects. They concluded that when the task entails shape-related judgments, attention selects from object-centered spaceinvariant representations. The rationale and results of Vecera and Farah's identification task experiments have been criticized elsewhere (see Kramer, Weber, \& Watson, 1997, and Vecera's, 1997, response; Driver \& Baylis, 1998).

In the last two experiments, Vecera and Farah (1994) used the same displays but modified the task so that subjects had to perform a cued detection task, instead of a shape judgment. On each trial, one of the two objects brightened briefly, cuing attention. Then the target, a small black dot, appeared on the contour of either the cued object (validcue trials) or the uncued object (invalid-cue trials). Subjects had to respond to the target onset. A cost in switching attention from the cued to the uncued object on invalidcue trials - that is, the object-based effect - was found when the two objects were distant from each other, but not when they were superimposed. Vecera and Farah concluded that attention operates on spatial unparsed representations when subjects must perform a simple detection task.

This conclusion is clearly at odds with other studies in the literature in which object-based effects were reported with detection tasks (e.g., Abrams \& Law, 2000; Egly, Driver, \& Rafal, 1994; Lamy \& Tsal, 2000). Note, however, that the absence of an object-based effect with Vecera and Farah's (1994) cued detection task may result from factors that have nothing to do with whether or not the task required shape judgments. The object-cuing procedure was such that the object contour thickened briefly. The target was a small dot of the same color as the object's contour on which it was superimposed. Thus, detection was likely to be harder when the target dot was on the cued object than when it was on the uncued object, because of forward masking. The fact that subjects tended to be slower in detecting the target when it appeared on the cued object than when it appeared on the uncued object is consistent with this forward-masking account.

In light of the above, there does not seem to be strong evidence in favor of Vecera and Farah's (1994) claim that object-based effects are to be found only when the task requires shape judgments.

\section{Object-Based Selection and the Spatial Extent of Attention}

Lavie and Driver (1996) suggested that the initial spatial setting of attention may influence object-based constraints on the distribution of attention. Their displays consisted of two dashed lines crossing each other in their middle and always contained two targets. Lavie and Driver found subjects to be slower at making same versus dif- ferent judgments when the two targets belonged to different objects than when they were located on the same object, even though the targets were equally distant from each other in the two conditions (Experiments 1-3). In Experiment 4, subjects were cued to attend to just one side of the display. Under these conditions of focused attention, object-based effects were no longer obtained. Lavie and Driver concluded that object-based effects may be found when attention is distributed in a diffuse mode across the display, but not when it is focused on a narrow spatial area within this display. This conclusion may apply only under specific circumstances yet to be defined, because there have been numerous reports of object-based effects with attention focused on a small part of the display (e.g., Atchley \& Kramer, 1998; Baylis \& Driver, 1992; Driver \& Baylis, 1989; Egly, Driver, \& Rafal, 1994; Kramer \& Jacobson, 1991). Moreover, Lamy (2000) reported two failures to replicate Lavie and Driver's finding (Experiment 4), using very similar displays.

So, at least on the basis of existing evidence, the spatial extent of attention does not seem to be a critical factor for defining the boundary conditions between space-based and object-based selection.

\section{Object-Based Attention and Object/Part Characteristics}

As we noted previously, the objects upon which selection occurs have usually been defined after the fact. That is, an object is most often a stimulus for which objectbased effects can be obtained and the objectness of which makes intuitive sense, on the basis of Gestalt principles of grouping. In order to tackle this problem, Watson and Kramer (1999) attempted to specify a priori the stimulus characteristics that define the objects upon which selection takes place. They proposed a framework that allows one to predict whether object-based effects will be found, depending on stimulus characteristics. Borrowing from Palmer and Rock's (1994) theory of perceptual organization, they distinguished between hierarchically organized levels of representation: (1) single uniformly connected (single-UC) regions, defined as connected regions with uniform visual properties, such as color or texture, and (2) grouped uniformly connected (grouped-UC) regions, which are larger representations made up of single-UC regions grouped on the basis of Gestalt principles. ${ }^{1}$

They used complex familiar objects (pairs of wrenches) and had subjects identify whether one or two predefined target properties were present in these objects. They examined under which conditions object-based effects - that is, performance costs for trials in which the two targets belong to different wrenches, rather than to the same wrenchcould be obtained for each representational level. They found (1) that object-based effects are obtained with singleUC objects (i.e., objects in which a target is not a singleUC region separate from the rest of the object to which it belongs), but not with grouped-UC objects (i.e., objects in which a target is a separate single-UC region), which led them to conclude that the default level at which selection 
occurs is the single-UC level, and (2) that selection may occur at the grouped-UC level when it is beneficial in order to perform the task or when this level has been primed. Figure 1 illustrates what the single-UC and grouped-UC levels refer to in the objects used in Watson and Kramer's (1999) study.

The finding that the level of representation at which objects are attended is flexible is consistent with earlier studies showing a role of top-down factors in object-based selection (e.g., Chen, 1998; Zemel, Behrmann, Mozer, \& Bavelier, 2000). Watson and Kramer's (1999) study adds a substantial contribution by proposing a framework that allows one to predict how predefined stimulus characteristics interact with such top-down factors as task demands and context.

Especially important, however, is the finding that one may predict a priori whether or not object-based effects will be found solely on the basis of object characteristics when there is no top-down bias toward selecting from grouped-UC representations. This finding suggests that attentional researchers may "no longer need to define objects available for selection in a post hoc manner but, instead, can begin to specify a priori the geometric and surface characteristics that define candidate objects" (Watson \& Kramer, 1999, p. 47). However, the conclusion that selection takes place at the single-UC level unless the task parameters encourage subjects to utilize grouped-UC representations is not readily reconciled with earlier research.

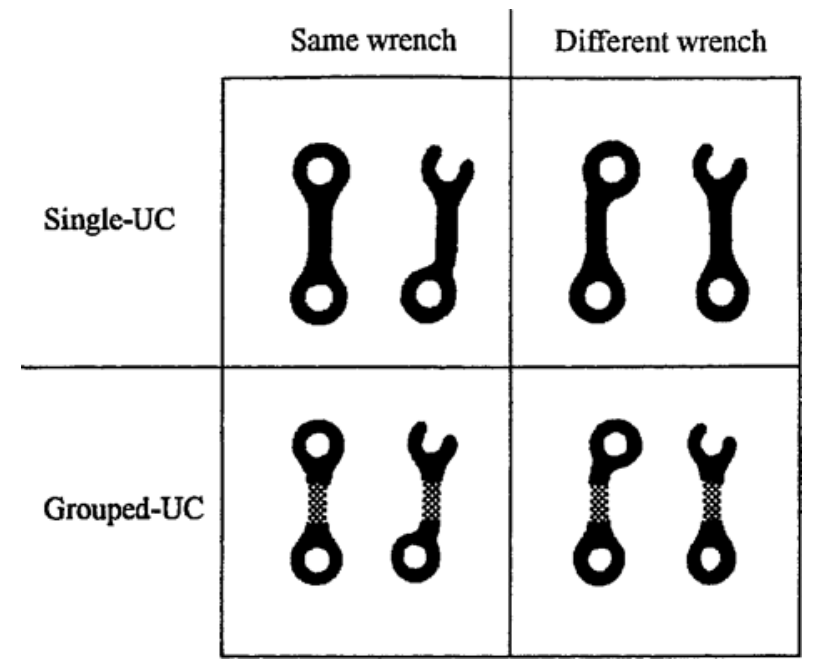

Figure 1. Examples of stimuli in Watson and Kramer's study (1999, Experiment 1). Subjects searched for the presence of two targets: an open wrench end and a bent wrench end. The lefthand panels illustrate same-object trials, in which the two targets belonged to the same wrench. The right-hand panels illustrate different-object trials, in which the two targets belonged to different wrenches. In the upper panels, the wrenches were uniformly gray. Thus, each wrench was a single uniformly connected (single-UC) region. In the lower panels, wrench ends were uniformly gray, but the handle between them was a red-and-blue checkerboard pattern. Thus, each wrench was a grouped-UC region, made up of three separate single- $\mathrm{U} C$ regions.
Egly, Driver, and Rafal (1994) had their subjects detect the onset of a white square at one of the four ends of two gray outline rectangles. One end was precued, as its borders whitened briefly. On valid-cue trials (80\% of the trials), the target appeared in the cued end of the cued rectangle, whereas on invalid-cue trials ( $20 \%$ of the trials), it appeared either in the uncued end of the cued rectangle or in the uncued rectangle. The distance between the cued location and the location where the target appeared was identical in both invalid-cue conditions. On invalid-cue trials, targets were detected faster when they belonged to the same object as the cue, rather than to the other object. Several replications have been reported with detection tasks (e.g., Abrams \& Law, 2000; Lamy \& Tsal, 2000; Vecera, 1994), as well as with identification tasks (e.g., Moore et al., 1998).

According to Watson and Kramer's (1999) classification, Egly, Driver, and Rafal's (1994) stimuli should be categorized as grouped-UC regions. The cue and the target constituted separate single-UC regions, since they were segregated from the rest of the object in which they appeared by a sharp boundary (white vs. black for the target and white vs. gray for the cue). Moreover, since on invalidcue trials, the target was equally likely to appear in the same object as the cue or in the alternative object, there was no obvious advantage in selecting from grouped-UC representations. Still, object-based effects were found. If the default selection level is the single-UC level, why did Egly, Driver, and Rafal's subjects select objects at the groupedUC level of representation?

Note that one may be intuitively reluctant to consider the stimuli used by Egly, Driver, and Rafal (1994) as being grouped-UC objects in the same sense as Watson and Kramer's (1999) objects. This is because in the former study, the objects initially appeared as single-UC regions, upon which a cue or a target were later superimposed, whereas in the latter, the targets appeared as intrinsic parts of the objects from the beginning of each trial. As a result, it is conceivable that in Egly, Driver, and Rafal's experiment, subjects were primed to process the cuing and target displays at the higher level of hierarchy represented by the rectangle including its contents (grouped-UC level) rather than at the lower level represented by the cue or the target (single-UC level). Consistent with this possibility, Watson and Kramer reported that "a prior selection episode at one level of object representation... can encourage the selection of subsequent objects at the same level of representation" (Experiment 4, p. 44). Although subjects were merely exposed to the rectangles prior to the target display, rather than having to select them, one may speculate that this prior exposure may explain why object-based effects were found in Egly, Driver, and Rafal's experiment. This point is addressed in Experiment 1. By way of a preview, the results do not support this speculation.

Moreover, it is not entirely clear whether, in Egly, Driver, and Rafal's (1994) study, the cue, the whitening of one of the rectangle's ends, was perceived to be a single-UC region, separate from the rest of the gray rectangle in which 
it appeared. If it was not perceived as a separate UC region, then in the cuing display, the whole UC region - that is, the whole rectangle-was selected as a result of cuing, which would explain why a target appearing in this object was responded to faster. Again by way of preview, this possibility can be dismissed on the basis of the results of Experiment 2 .

There are numerous other differences between Watson and Kramer's (1999) study and that of Egly, Driver, and Rafal (1994). For instance, the studies differed in the use of complex realistic stimuli versus simple geometric shapes, a shape discrimination task versus a simple detection task, and a divided-attention paradigm versus a spatial-cuing paradigm. However, if the notion of UC regions was sufficient to predict when object-based effects should be found in tasks involving no top-down bias toward selection at the grouped-UClevel, each of these factors should be irrelevant. Thus, the discrepancy between the two studies suggests that other factors may interact with stimulus characteristics to determine how object structure affects the distribution of attention.

The objective of this paper was to identify the conditions necessary to obtain object-based effects with objects that can be classified as grouped-UC regions according to Watson and Kramer's (1999) terminology. We used the divergence between Watson and Kramer's results and those of Egly, Driver, and Rafal (1994) as a starting point in our attempt to explore this question. In all four experiments presented below, stimulus factors were kept as constant as possible, while task characteristics were systematically manipulated. The stimuli were grouped-UC objects similar to those used by Egly, Driver, and Rafal.

In Experiment 1, subjects performed a same versus different judgment task. Thus, as in Watson and Kramer's (1999) experiments, they had to attend to two simultaneous targets embedded in objects. In Experiment 2, a spatialcuing paradigm was used, similar to that of Egly, Driver, and Rafal (1994). The to-be-judged targets were identical in the two experiments and the two tasks required making a size discrimination. By way of a preview, object-based effects were obtained in Experiment 2 (replicating Egly, Driver, and Rafal's, 1994, finding), but not in Experiment 1 (replicating Watson and Kramer's, 1999, finding). The objective of Experiments 3 and 4 was to determine why these two tasks yielded different results.

\section{EXPERIMENT 1}

Before attempting to elucidate what task differences may have caused the disagreement between the two sets of studies, it is important to ensure that the absence of an object-based effect reported by Watson and Kramer (1999) for grouped-UC objects (in their Experiment 1) is a general phenomenon and can be replicated with stimuli similar to those of Egly, Driver, and Rafal (1994).

Displays consisted of two outline rectangles. On each trial, two targets appeared, each of which was either a large or a small filled square located at an end of one of the rectangles. Subjects had to judge whether the targets were the same or different with respect to size. In this experiment, object-based effects would be revealed as better performance when the two targets appear in the same, rather than in different, objects.

\section{Method}

Subjects. The subjects were 11 undergraduate students at Johns Hopkins University, who participated in the experiment for course credit. All had normal or corrected-to-normal vision.

Apparatus and Stimuli. The stimuli were presented on an IBMcompatible PC attached to a VGA color monitor. The fixation display consisted of a central $0.4^{\circ} \times 0.4^{\circ}$ plus sign $(+)$ and two outline rectangles, each subtending $11^{\circ}$ in height and $2.2^{\circ}$ in width. The rectangles were either vertically oriented, to the left and right of fixation, or horizontally oriented, above and below fixation. Stroke width was $0.1^{\circ}$. The rectangles were equally distant from the fixation sign, and their center-to-center distance was $8.8^{\circ}$. The overall display subtended $11^{\circ} \times 11^{\circ}$. In the target display, two targets were simultaneously superimposed on two of the rectangles' ends. They never occupied diagonally opposed locations. Each target was a filled square, which was either small $\left(1.4^{\circ}\right)$ or large $\left(2.2^{\circ}\right)$. The fixation sign, rectangles, and targets were light gray and were drawn on a black background.

Procedure. The subjects were tested in a dimly lit, sound-attenuated room. Viewing distance was maintained at $60 \mathrm{~cm}$ from the computer monitor, using a chinrest. The sequence of events in each trial is illustrated in Figure 2. At the beginning of each trial, the fixation display was shown for $1,000 \mathrm{msec}$ and was equally often oriented vertically or horizontally. Thus, each of the objects first appeared as a single-UC region. Then the target display appeared and remained visible until the subject responded. Thus, in the target display, the objects were grouped-UC regions. The screen went blank for $500 \mathrm{msec}$ before the next trial began.

The subjects' task was to press " 1 " if the targets were the same (both small or both large) and " 2 " if they were different (one small and one large), using the numerical keypad with their dominant hand. They were instructed to respond as quickly as possible, because response latency (RT) was recorded, and also to minimize the number of errors. A 500-msec feedback beep was sounded if a subject made an error or an anticipatory response $(\mathrm{RT}<150 \mathrm{msec})$. The subjects were asked to maintain fixation throughout each trial.

After the subjects heard the instructions, they were given 20 practice trials randomly selected from the experimental trials. The order of the trials was randomized by computer for each subject. There were six blocks of 96 experimental trials each. The subjects were allowed a rest period between them.

Same and different trials were equally probable and were presented in randomized order. In the same condition, the targets were equally likely to be small or large. The targets appeared equally often within the same object or within different objects. Orientation was not included as a factor in the experimental design, because in previous studies that used similar stimuli and procedures (Egly, Driver, \& Rafal, 1994; Lamy \& Tsal, 2000; Vecera, 1994), this variable was found not to be a significant source of variance. All the conditions were randomly intermixed.

\section{Results}

Accuracy. Mean accuracy was 93\%. An analysis of variance (ANOVA) was conducted, with same versus different object and same versus different response as factors. There was no main effect of either same versus different object $[93 \%$ vs. $92 \%$, respectively; $F(1,10)=1.44$, 


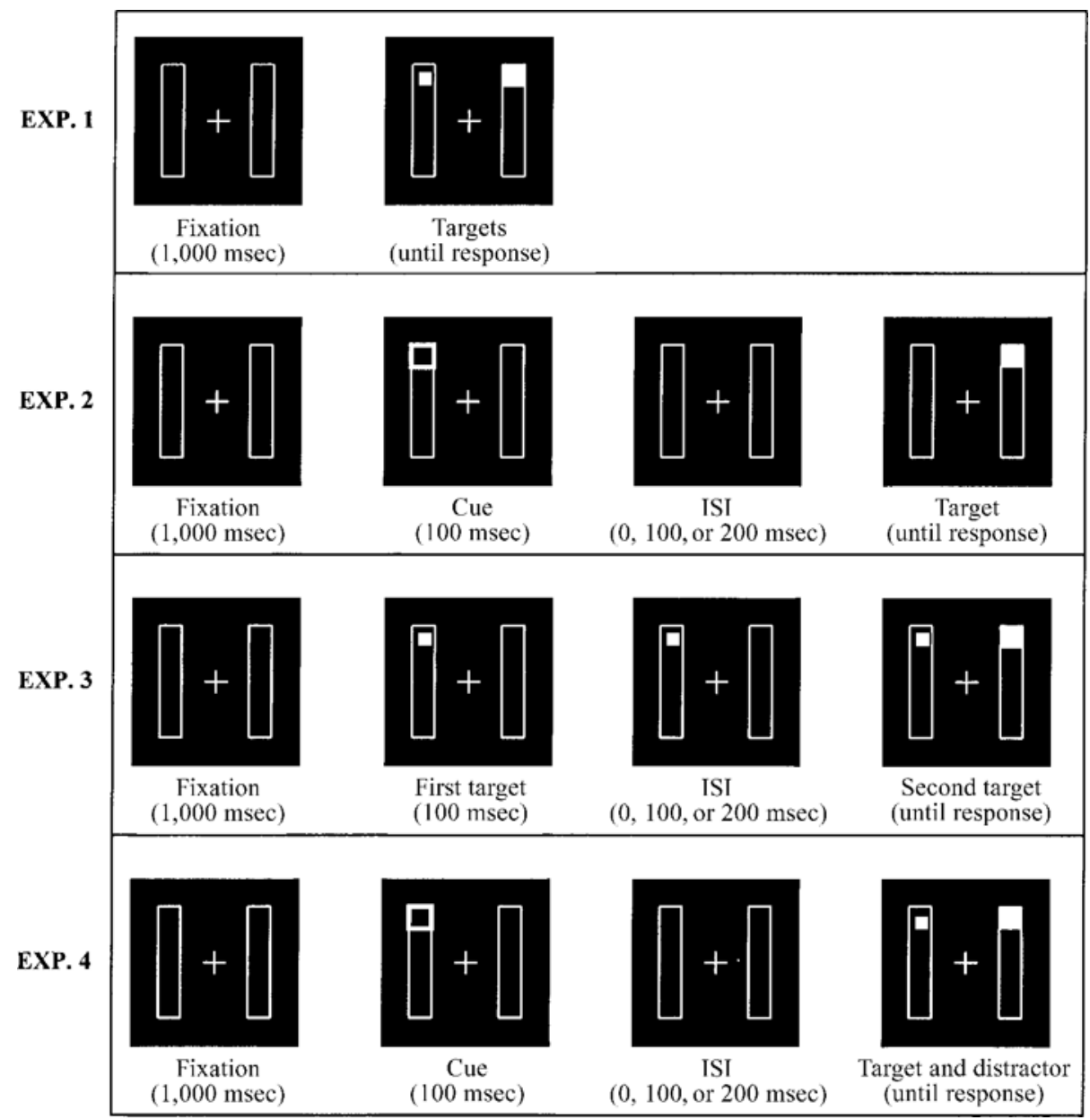

Figure 2. Stimuli and sequence of events in Experiments 1-4. The examples correspond to the different-object condition and, where relevant, to the different-response condition.

$p>.3$ ] or same versus different response [93\% vs. $92 \%$, respectively; $F<1]$. The interaction between the two factors was nonsignificant $(F<1)$. Thus, speed-accuracy tradeoff was not a concern.

Reaction times. Error trials were removed from analysis. RTs for each subject were sorted into cells according to the conditions of same versus different object and same versus differentresponse. The RTs exceeding the cell mean by more than 2.5 standard deviations were trimmed. This removed fewer than $0.5 \%$ of the observations.

Mean RTs for correct trials are presented in Figure 3. An ANOVA was conducted, with same versus different object and same versus different response as factors. There was no main effect of same versus different object (592 vs. $590 \mathrm{msec} ; F<1)$ and no main effect of same versus different response [583 vs. $599 \mathrm{msec} ; F(1,10)=2.01, p>.1]$. The interaction between the two factors approached significance $[F(1,10)=4.44, p<.07]$. Post hoc analyses indicated that when the targets were the same, responses tended to be faster in the different- than in the same-object condition [579 vs. $587 \mathrm{msec} ; F(1,10)=3.53, p<.09]$. The difference between same- and different-object trials did not approach significance in the different-response condition [597 vs. $601 \mathrm{msec} ; F(1,10)=1.89, p>.1]$.

\section{Discussion}

In this experiment, we used grouped-UC stimuli similar to those of Egly, Driver, and Rafal (1994). The task was similar to Watson and Kramer's (1999) in that it required processing two targets that appeared simultaneously in locations that belonged either to the same or to different objects. There was no object-based effect. The results of the present experiment allow us to reject several of the differences that were suggested earlier as potential sources for the discrepant findings reported by Egly, Driver, and Rafal versus Watson and Kramer. These include stimulus complexity and the use of a discrimination versus a detection task. Moreover, the fact that the objects were initially pre- 

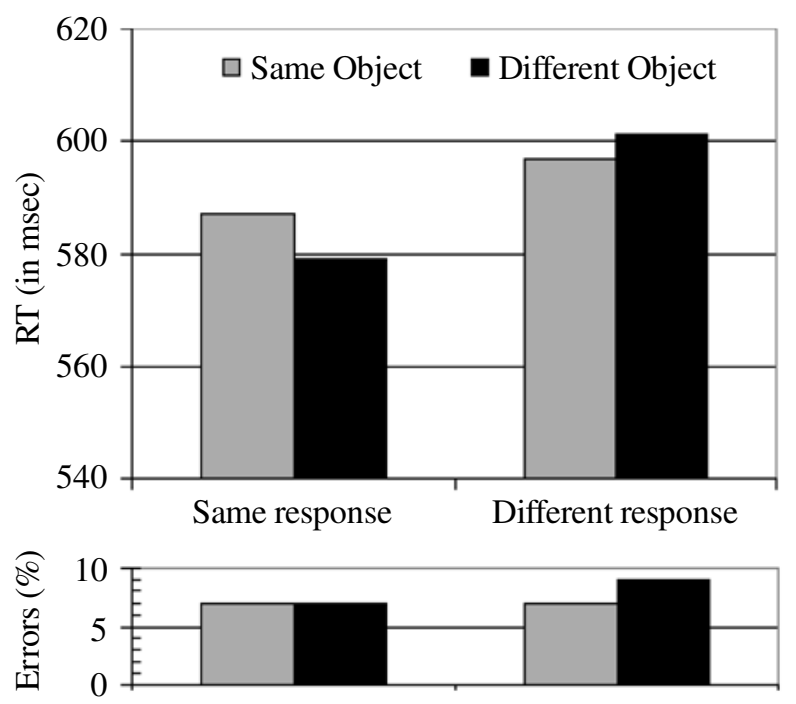

Figure 3. Experiment 1: Mean reaction times (RTs) in milliseconds and percentage (\%) of errors to make same versus different judgments in the same-object and different-object conditions.

sented as single-UC regions on which the targets were later superimposed did not encourage subjects to select from grouped-UC representations.

Thus, Watson and Kramer's (1999) finding was replicated. We next attempted to determine whether objectbased effects would be obtained using Egly, Driver, and Rafal's (1994) procedure with the same apparatus and stimuli. This was the objective of Experiment 2.

\section{EXPERIMENT 2}

Although the results obtained by Egly, Driver, and Rafal (1994) are often cited as evidence for object-based selection, the mechanism that causes faster responses for sameobject targets remains unspecified. Two different possibilities have often been suggested (e.g., Davis, Driver, Pavani, \& Shepherd, 2000; Driver \& Baylis, 1998), but there has been no empirical attempt to disentangle them.

On the one hand, the object-based effect found in Egly, Driver, and Rafal's (1994) study may stem from a greater difficulty in shifting attention between objects than within objects over a comparable distance. On the other hand, this effect may indicate that attention tends to spread across the cued object before the target appears, even though the best strategy would seem to be for subjects to remain with their attention tightly focused on the most probable target location until the target appears. Thus, according to this account, attention propagates passively within the cued object - that is, independently of the subjects' intentionsas a consequence of being focused on part of an object. In Watson and Kramer's (1999) task, there was no such opportunity for attention to spread, because the two targets appeared simultaneously and there was no precue. This could explain why no object-based effects were obtained.
In this experiment, we investigated whether in Egly, Driver, and Rafal's (1994) spatial-cuing paradigm, attention spreads to object parts grouped with the cued location. To explore this issue, the interval between cue onset and target onset (cue-to-target stimulus onset asynchrony [SOA]) was manipulated. If attention spreads within the cued object before the target appears, the object-based effect is expected to be smaller at shorter SOAs. It was also important to ensure that, using the same targets and objects as those in Experiment 1, we could replicate Egly, Driver, and Rafal's results. This experiment was similar to Egly, Driver, and Rafal's, except for three differences. We used three cue-to-target SOAs (100, 200, and $300 \mathrm{msec})$ instead of just one $(300 \mathrm{msec})$. In addition, the subjects had to categorize the target as being small or large, rather than simply detecting its presence. Finally, the cue was a red outline square instead of the whitening of one of the rectangles' ends, so that it would be unambiguously perceived as a separate single-UC region.

\section{Method}

Subjects. The subjects were 12 undergraduate students at Johns Hopkins University, who participated in the experiment for course credit. All had normal or corrected-to-normal vision.

Apparatus, Stimuli, and Procedure. This experiment was similar to Egly, Driver, and Rafal's (1994). The apparatus and stimuli were identical to those used in Experiment 1, except for the following changes. The sequence of events in each trial is illustrated in Figure 2. A cuing display followed the fixation display. It consisted of a cue superimposed on the fixation display. The cue was a red outline $2.2^{\circ} \times 2.2^{\circ}$ square superimposed on the end of one of the shapes. It appeared for $100 \mathrm{msec}$, equally often in each of the four ends. The target display was presented immediately at cue offset, after $100 \mathrm{msec}$ or after $200 \mathrm{msec}$. Thus, the cue-to-target SOA was 100, 200, or $300 \mathrm{msec}$. In the target display, one target, equally often small or large, was superimposed on the stimulus display, also at one of the rectangles' ends. No target appeared on catch trials, in which the target display was therefore identical to the fixation display.

The subjects" task was to press " 1 " if the target was small and " 3 " if the target was large, using the numerical keypad with their dominant hands. They were asked to pay attention to the cue, because it specified the location at which the target was most likely to appear. They were also told that on a small proportion of the trials, the target would appear in an uncued location. Each of the six blocks included 128 experimental trials.

Design. The experiment was a within-subjects design. The target appeared at the cued end on $80 \%$ of the trials (valid-cue condition) and at an uncued end on $20 \%$ of the trials (invalid-cue condition). In the latter condition, it appeared equally often at the uncued end of the cued rectangle and at the equidistant end of the uncued rectangle. In order to keep cue-to-target distance constant across invalidcue conditions, the target never appeared at the location diagonal to the cued location. The three cue-to-target SOA conditions were equiprobable within both the valid- and the invalid-cue conditions. All the conditions were randomly intermixed.

\section{Results}

Accuracy. Mean accuracy was 98\%. The first ANOVA was conducted with validity (valid- vs. invalid-cue trials), SOA (100, 200, and $300 \mathrm{msec}$ ), and target size (small vs. large) as factors. No effect reached significance. An additional ANOVA was conducted on invalid-cue trials, with 

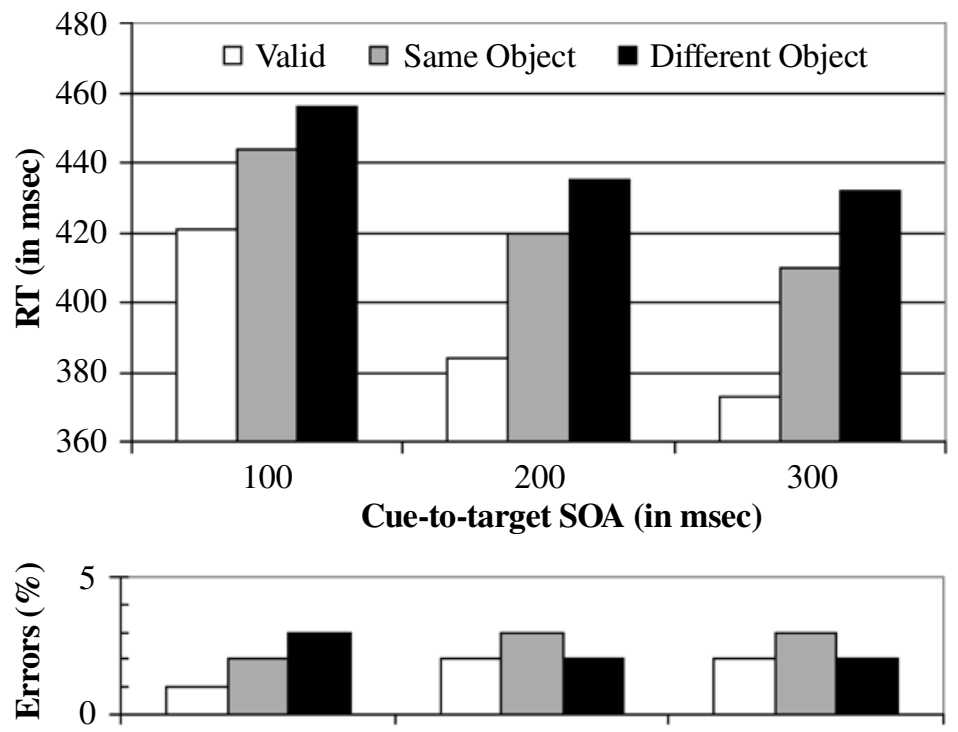

Figure 4. Experiment 2: Mean reaction times (RTs) in milliseconds and percentage $(\%)$ of errors to categorize the target in the valid-cue, invalid-cue sameobject, and invalid-cue different-object conditions, as a function of cue to target SOA.

same versus different object, SOA, and target size as factors. The effect of target size was significant $[99 \%$ vs. $97 \%$ for the small vs. the large target, respectively; $F(1,11)=$ $5.17, p<.05]$. No other effect approached significancenamely, no effect involving the same versus different object factor $($ all $F \mathrm{~s}<1)$. Thus, speed-accuracy tradeoff was not a concern.

Reaction times. Error trials were removed from analysis. RTs for each subject were sorted into cells according to the conditions of validity, SOA, and target size. RTs exceeding the cell mean by more than 2.5 standard deviations were trimmed. This removed fewer than $0.5 \%$ of the observations.

Mean RTs for correct trials are presented in Figure 4. In order to assess purely space-based effects, the first ANOVA was conducted with validity (valid-cue trials vs. invalidcue same-object trials), SOA (100, 200, and $300 \mathrm{msec}$ ), and target size (small vs. large) as factors.

The subjects were faster to respond on valid-cue than on invalid-cue same-object trials [393 vs. $425 \mathrm{msec}$; $F(1,11)=39.98 ; p<.0001]$. The main effect of SOA was also significant $[F(2,22)=109.02, p<.0001]$. Post hoc comparisons showed that the subjects were faster to respond in 300-msec than in 200-msec SOA trials [379 vs. $390 \mathrm{msec} ; F(1,11)=11.45, p<.007]$ and were faster on 200 -msec than on 100 -msec SOA trials [390 vs. $425 \mathrm{msec}$; $F(1,11)=116.63, p<.0001]$. There was no main effect of target size $(398 \mathrm{msec}$ for small targets vs. $399 \mathrm{msec}$ for large targets; $F<1$ ).

There was a significant interaction between validity and SOA $[F(2,22)=4.27, p<.03]$. Post hoc comparisons showed that validity effects were smaller on $100-\mathrm{msec}$ SOA trials than on 200- and 300-msec SOA trials [23 vs.
36 and $37 \mathrm{msec} ; F(1,11)=6.98, p<.03]$. However, the cuing advantage was still significant in the $100-\mathrm{msec}$ SOA condition $[F(1,11)=22.77, p<.0006]$.

Validity effects were also larger when the target was large than when it was small [24 vs. $39 \mathrm{msec} ; F(1,11)=$ $6.14, p<.03]$. No other interaction reached significance.

In order to assess object-based effects, an additional ANOVA was conducted on invalid-cue trials, with same versus different object, SOA, and target size as factors. The subjects were faster to respond when the target appeared within the same object as the cue than within the other object [ 425 vs. $441 \mathrm{msec} ; F(1,11)=24.58, p<.0004$ ]. The main effect of SOA was also significant $[F(2,22)=$ $33.53, p<.0001]$. Post hoc comparisons showed that the subjects were faster on 300-msec than on 200-msec SOA trials [ $421 \mathrm{vs.} 428 \mathrm{msec} ; F(1,11)=4.88, p<.05]$ and also were faster on 200-msec than on 100-msec SOA trials [428 msec vs. $450 \mathrm{msec} ; F(1,11)=28.24, p<.0002$ ]. There was no main effect of target size (404 msec for the small target vs. $405 \mathrm{msec}$ for the large target; $F<1$ ). No interaction reached significance. In particular, same versus different object and SOA did not interact $[F(2,22)=$ $1.34, p>.2]$.

\section{Discussion}

In this experiment, both the space-based and the objectbased effects obtained by Egly, Driver, and Rafal (1994) were replicated.

The SOA manipulation affected the space-based effect. Specifically, the cuing advantage (i.e., the difference between valid- and invalid-cue trials) was significant when the target appeared $100 \mathrm{msec}$ after cue onset, but it was smaller than at longer SOAs. This finding is consistent 
with the results reported by Eriksen and his colleagues (Eriksen \& St. James, 1986; Eriksen \& Yeh, 1985). They showed that subjects are unable to focus their attention optimally on a precued location at cue-to-target SOAs shorter than $100 \mathrm{msec}$ and that distractors surrounding the target can be ignored more efficiently as SOAs become longer. They suggested that it takes some time for attention to become focused on a restricted area.

In contrast, the magnitude of the object-based effect (i.e., the difference between the same- and the differentobject conditions on invalid-cue trials) was unaffected by the SOA manipulation. This finding suggests that attention does not spread to locations within the cued object before the target appears, because if it did, one would expect the object-based effect to become larger as the time available for attention to propagate from the cued location to other locations within the same object becomes longer. One may argue that if it takes attention less than $100 \mathrm{msec}$ to fully propagate within the cued object, our procedure may not have been sensitive enough to capture the phenomenon. Countering this, however, we have also shown that a $100-\mathrm{msec}$ SOA is not enough for attention to become fully focused on the cued location. It would be useful to further confirm this interpretation by testing even shorter SOAs.

Three main conclusions can be drawn from the results of the present experiment. First, we were able to replicate Egly, Driver, and Rafal's (1994) findings, using the same objects and targets and the same apparatus as those in Experiment 1, where no object-based effects were obtained. This confirms our hypothesis that, for reasons yet to be elucidated, object-based effects are found with groupedUC objects, using a spatial-cuing paradigm, but not using a divided-attention paradigm.

Second, the idea that attention propagates within the cued object during the cue-to-target SOA, which is often cited as a possible account for Egly, Driver, and Rafal's (1994) finding, proves wrong when tested empirically. It is thus more likely that Egly, Driver, and Rafal's results reveal a cost of shifting attention between objects.

Third, since Experiment 2 showed that the propagation of attention within the cued object is not necessary for obtaining an object-based effect, the fact that there was no opportunity for attention to propagate in Experiment 1 cannot explain why this experiment did not yield object-based effects. In the following two experiments, we shall attempt to test alternative accounts for the finding that objectbased selection with grouped-UC objects was revealed in a spatial-cuing task, but not in a divided-attention task.

\section{EXPERIMENT 3}

In interpreting the absence of object-based effects with grouped-UC displays, Watson and Kramer (1999) assumed that an attentional shift was necessary whether the target properties appeared on a single wrench or on two different wrenches, because in both cases, attention had to be shifted between two different UC regions. However, one may consider an alternative interpretation of these results.
Note that the task employed by Watson and Kramer was to decide whether one or two targets were present in each display. Thus, their task involved counting the targets, rather than comparing them. In conditions in which the targets formed separate single UC regions, these may have been distinct enough to allow subjects to treat the task as a subitizing task. Since subitizing - that is, the enumeration of a small number of items - can be performed in parallel (e.g., Trick \& Pylyshyn, 1993), the subjects in Watson and Kramer's study may have processed the two targets in parallel, with no need to shift their attention from the one to the other.

In Experiment 1 of the present paper, the subjects had to judge whether the two targets were the same or different. Note that the stimuli used were such that if the targets were the same, displays were symmetrical relative to either the vertical or the horizontal axis, whereas if the targets were different, displays were asymmetrical. Humans' particular sensitivity to visual symmetry has been richly documented. For instance, Pascal asserted that "symmetry is what you see at a glance" (Pascal, cited by Baylis \& Driver, 1994, p. 377). Thus, it is conceivable that instead of comparing the targets' sizes, as the task explicitly required, the subjects used the alternative strategy of responding same when they detected symmetry and different when they did not. Baylis and Driver (1994) found that "symmetry within a shape can be detected in parallel, at least when potential axes of symmetry are restricted to known possibilities"(p. 396). In our first experiment, the subjects could judge the symmetry of each display as a whole along either the vertical or the horizontal axis in order to perform the task. According to Baylis and Driver's (1994) finding, this could be done without having to shift their attention between the two targets.

On invalid-cue trials in Experiment 2, as in Egly, Driver, and Rafal's (1994) experiment, attention had to be redirected from the invalidly cued location, on which it was focused at the time the target appeared, to the target location inside either the same or a different object. Thus, the task involved shifts of attention within and between objects.

The foregoing observations suggest the possibility that the critical difference between Experiments 1 and 2 (and between Watson and Kramer's 1999, and Egly, Driver, and Rafal's, 1994, studies) resides in whether or not the task required shifts of attention. In other words, we propose that with grouped-UC objects, shifts of attention may be a necessary condition for object-based effects to be observed.

This hypothesis is based on the speculation that the two divided-attention studies in which no object-based effects were obtained (Watson \& Kramer's, 1999, and the present Experiment 1) could be regarded as special cases. Specifically, we assumed in a post hoc manner that, in these studies, subjects had the opportunity to use alternative strategies, whereby the task could be performed in parallel and without shifting attention between the two targets.

The objective of the next two experiments was to test our hypothesis by designing tasks in which there could be no ambiguity as to whether or not shifts of attention were 
required. In Experiment 3, the task was such that the subjects had to shift their attention, whereas in Experiment 4, the subjects were induced to avoid making such shifts.

Experiment 3 was identical to Experiment 1, except that the targets appeared one at a time, instead of simultaneously. This procedure forced the subjects to shift their attention between the targets and prevented them from selecting them simultaneously. We reasoned that if object-based effects result from the need for an attentional shift, objectbased effects should be observed in this experiment.

\section{Method}

Subjects. The subjects were 10 undergraduate students at Johns Hopkins University, who participated in the experiment for course credit. All had normal or corrected-to-normal vision.

Apparatus, Stimuli, Procedure, and Design. The sequence of events is illustrated in Figure 2. The apparatus, stimuli, and procedure were identical to those used in Experiment 1, except for the following change. Instead of being presented simultaneously, the targets appeared with a variable SOA of 100,200 , or $300 \mathrm{msec}$. SOA conditions were equiprobable and were presented in randomized order.

\section{Results}

Accuracy. Mean accuracy was $96 \%$. An ANOVA was conducted, with same versus different object, same versus different response, and SOA as factors. No effect approached significance (all $F_{\mathrm{S}}<1$ ). Thus, speed-accuracy tradeoff was not a concern.

Reaction times. Error trials were removed from analysis. RTs for each subject were sorted into cells according to the conditions of same versus different object, same versus different response, and SOA. RTs exceeding the cell mean by more than 2.5 standard deviations were trimmed.
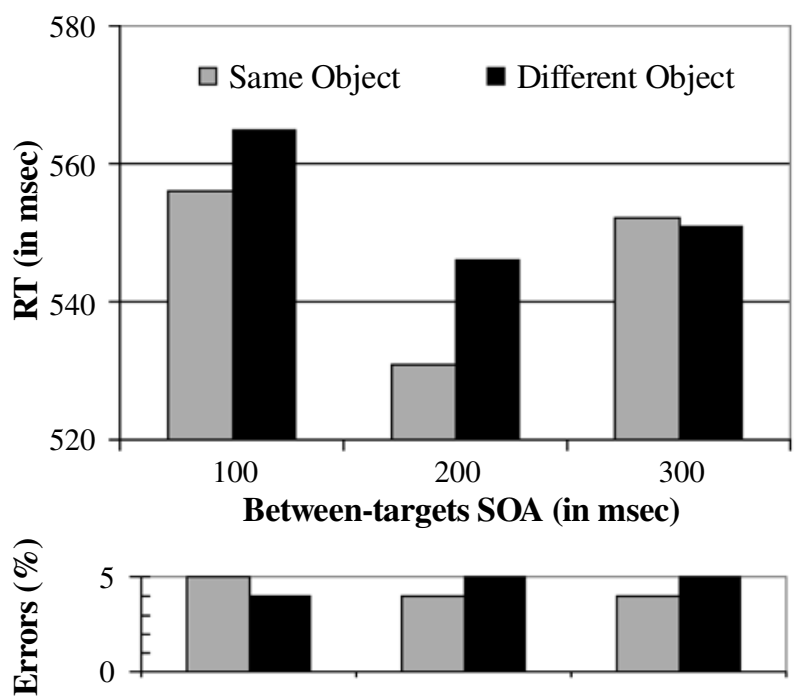

Figure 5. Experiment 3: Mean reaction times (RTs) in milliseconds and percentage (\%) of errors to make same versus different judgments in the same-object and different-object conditions as a function of between-targets stimulus onset asynchronies (SOAs).
This removed fewer than $0.5 \%$ of the observations. Mean RTs for correct trials are presented in Figure 5. An ANOVA was conducted, with same versus different object, same versus different response, and SOA as factors. There was an object-based effect, revealed in faster responses when the two targets were in the same object rather than in different objects [546 vs. $554 \mathrm{msec} ; F(1,9)=6.62, p<.03$ ] The effect of SOA was also significant $[F(2,18)=12.84$, $p<.0003$ ]. Post hoc comparisons revealed that the subjects were fastest when the two targets were onset at an interval of $200 \mathrm{msec}$ [ $538 \mathrm{vs} .561 \mathrm{msec}$ and $552 \mathrm{msec}$, for the 100- and 300-msec SOAs, respectively; $F(1,9)=$ $28.69, p<.0005]$. Same responses were faster than different responses [540 vs. $560 \mathrm{msec} ; F(1,9)=6.17, p<.04$ ]

The interaction between SOA and same versus different object was significant $[F(2,18)=4.88, p=.02]$. The same versus different object effect was significant at both the 100 - and the 200-msec SOAs [ 9 msec, $F(1,9)=10.18, p<$ .01 , and $15 \mathrm{msec}, F(1,9)=13.06, p<.006$, respectively], with a similar magnitude in the two conditions $[F(1,9)=$ $1.63, p>.1]$. In contrast, there was no same versus different object effect at the 300 -msec SOA (552 vs. $551 \mathrm{msec}$; $F<1)$.

\section{Discussion}

The objective of this experiment was to investigate the idea that an attentional shift between objects may be the necessary condition to obtain object-based effects with grouped-UC objects. The task, targets, and objects were the same as those in Experiment 1, but the subjects had to shift their attention between the two targets in order to perform the task. In sharp contrast with the null result found in Experiment 1, object-based effects were obtained under these conditions.

The present experiment yielded an additional finding worth noting. The object-based effect was modulated by the interval between the two targets, whereas in Experiment 2 , this effect was not influenced by the cue-to-target interval. Note that the events that are likely to have taken place during the SOA are very different in the two experiments. In Experiment 2, the target appeared at the cued location most of the time. This should have encouraged the subjects to maintain their attention focused on that location throughout the cue-to-target interval. When the target appeared at an invalid location, they had to shift their attention to its location, but this happened only after the SOA had elapsed. Thus, in Experiment 2, the SOA could not interact with whether or not the subjects had to shift their attention. In contrast, in Experiment 3, the best strategy was for the subjects to redistribute their attention broadly, immediately after processing the first target. According to this logic, attention was initially summoned to the location of the first onset target, and it took between 200 and $300 \mathrm{msec}$ for attention to become maximally distributed. It follows that, whereas at the shorter SOAs, attention had to be shifted to the second target, at the 300msec SOA, no shift of attention was required. The latter condition was similar to the situation prevailing in Exper- 
iment 1 . The way the length of the target-to-target SOA modulated the object-based effect in this experiment is thus consistent with the idea that object-based effects result from a cost in shifting attention between objects.

\section{EXPERIMENT 4}

Experiment 3 was designed to force subjects to shift their attention between two targets that belonged either to the same or to different objects. In line with our predictions, under these conditions, object-based effects were obtained. In contrast, the present experiment was designed to encourage subjects not to shift their attention and to maintain it focused on a precued location. The objective of this experiment was to show that when no shift of attention is required, no object-based effects are found.

We used a variant of Eriksen's (Eriksen \& Hoffman, 1973) flanker task. A cuing display summoned the subject's attention to the cue's location, where the target appeared on $100 \%$ of the trials. Then the target and one distractor appeared simultaneously, either within the same object or within different objects. The distractor had either the same size as the target (compatible condition) or the alternative size (incompatible condition). The subjects had to judge the target's size, while ignoring the distractor. It was expected that the subjects would be faster to respond when the target and the distractor were the of same size than when they were of different sizes. If selection is affected by the grouped-UC level of organization in this experiment, such compatibility effects should be larger when the target and the distractor appear in the same object. Because compatibility effects may not be obtained when the target and the distractor are separated by a large distance (e.g., Eriksen \& St. James, 1986), as was the case in our displays, we also included a condition in which no distractor appeared. This allowed us to measure interference effects - that is, the extent to which the mere presence of the distractor (rather than its identity) interfered with performance. Again, object-based effects would be revealed as higher costs of distractor presence when the distractor appeared in the same object as the target.

According to our hypothesis, no object-based effects should be found in this experiment, because subjects did not have to shift their attention.

\section{Method}

Subjects. The subjects were 10 undergraduate students at Johns Hopkins University, who participated in the experiment for course credit. All had normal or corrected-to-normal vision.

Apparatus, Stimuli, Procedure, and Design. The sequence of events is illustrated in Figure 2. The apparatus, stimuli, and procedure were identical to those used in Experiment 1, except for the following changes. After the fixation display, the same cuing display as that in Experiment 2 appeared for $100 \mathrm{msec}$. In the target display, the target appeared at the cued location on $100 \%$ of the trials, after an SOA of 100, 200, or $300 \mathrm{msec}$. On $67 \%$ of the trials, an additional filled square appeared simultaneously with the target (distractorpresent condition). On the remaining 33\% of the trials, only the target was presented (distractor-absent condition).
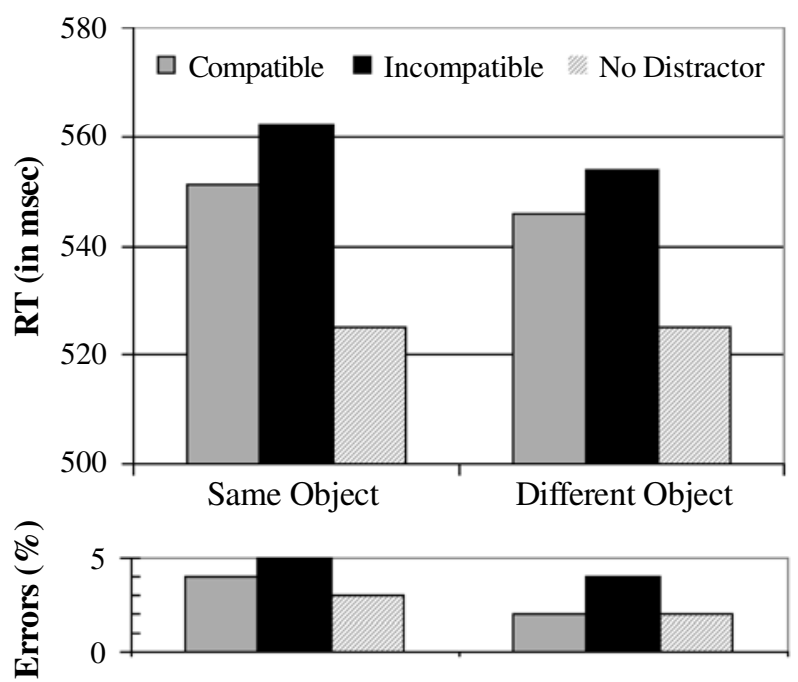

Figure 6. Experiment 4: Mean reaction times (RTs) and percentage $(\%)$ of errors in milliseconds on no-distractor and on compatible- and incompatible-distractor trials in the same-object and different-object conditions.

In the distractor-present condition, the distractor appeared equiprobably at the uncued location within the same object as the target (same-object condition) and at an equidistant location within the other object (different-object condition). It never appeared in the location diagonally opposite the target location. The distractor was equally likely to be identical to the target (compatible-distractor condition) or to have the alternative size (incompatible-distractor condition). All the conditions were presented in randomized order.

\section{Results}

Accuracy. Mean accuracy was 97\%. An ANOVA was conducted, with same versus different object, same versus different response, and SOA as factors. No effect approached significance. Thus, speed-accuracy tradeoff was not a concern.

Reaction times. Error trials were removed from analysis. For each ANOVA, RTs for each subject were sorted into cells according to the analysis factors. RTs exceeding the cell mean by more than 2.5 standard deviations were trimmed. This removed fewer than $0.5 \%$ of the observations for both analyses.

Mean reaction times for correct trials are presented in Figure 6. A first ANOVA was conducted, with distractor presence and SOA as factors. There was a significant interference effect, revealed in slower responses when the distractor was present rather than absent [ $550 \mathrm{vs} .525 \mathrm{msec}$; $F(1,9)=29.47, p<.0004]$. The effect of SOA was also significant $[F(2,18)=15.71, p<.0001]$, with slower responses at the shortest SOA [562 msec vs. 530 and $533 \mathrm{msec}$, for the 100-msec SOA vs. the 200- and 300-msec SOAs, respectively; $F(1,9)=26.89, p<.0006]$. The interaction between the two factors did not reach significance $[F(2,18)=$ $1.10, p>.3]$.

A second ANOVA was conducted on distractor-present trials, with same versus different object, compatible versus 
incompatible distractor, and SOA as factors. There was a significant effect of SOA $[F(1,18)=7.77, p<.004]$, with slower responses at the shortest SOA [571 msec vs. 541 and $548 \mathrm{msec}$, for the $100-\mathrm{msec}$ SOA vs. the 200- and 300 -msec SOAs, respectively; $F(1,9)=11.74, p<.008$ ]. Responses were faster in the compatible- than in the incompatible-distractor condition [548 vs. $558 \mathrm{msec}$; $F(1,9)=6.00, p<.04]$. There was no same- versus differentobject effect [557 vs. $550 \mathrm{msec} ; F(1,9)=1.38, p>.2]$. There was no significant interaction. Specifically, compatibility and same- versus different-object conditions did not interact $(F<1)$.

\section{Discussion}

The results of this experiment showed that the presence of a distractor interfered with the subjects' performance in judging the size of a target that appeared at a precued location. However, there was no object-based modulation of this interference effect. Moreover, responses were faster when the size of the distractor was compatible with the response elicited by the target than when it was incompatible with it. Again, this flanker effect was of the same magnitude whether or not the target and the distractor belonged to the same object. These results are consistent with the claim that when the task does not require a shift of attention, no object-based effects are obtained.

\section{GENERAL DISCUSSION}

The objective of this paper was to define under what conditionsobject-based effects are observed. Recently, Watson and Kramer (1999) provided novel evidence in support of Palmer and Rock's (1994) idea that the principle of uniform connectedness plays a fundamental role in perceptual organization. They showed that object-based effects, revealed as a difficulty in judging two attributes that belonged to different objects, were obtained only when these objects were defined as single-UC regions. They also showed that object-based effects with objects defined at a higher level of hierarchy - that is, the grouped-UC level-were observed only if top-down factors biased attentional selection to occur at that level. Thus, Watson and Kramer proposed a framework that allows one to predict whether or not object-based effects will be found, depending on well-defined stimulus characteristics, under conditions in which top-down factors do not induce a bias toward selection at the grouped-UC region level.

The results of the four experiments presented here indicate that the framework proposed by Watson and Kramer (1999) does not suffice to delineate the boundary conditions of object-based selection. Taken together, our results suggest that the grouped-UC level of perceptual organization does not affect performance, in line with Watson and Kramer's findings, but only when no shift of attention is required. Indeed, we showed that when attention needs to be shifted from one single-UC region to another, performance is better when the two single-UC regions belong to the same perceptual group (or grouped-UC region)- that is, object-based effects are observed, in contrast with Watson and Kramer's predictions.

In all four experiments, we used the same object structure-namely, the target (white filled square) and, when present, the cue (red outline square) were single-UC regions embedded within one of two grouped-UC regions (white outline rectangles). The nature of the task (size discrimination) was also kept constant across experiments.

In Experiment 1, the subjects performed a task similar to Watson and Kramer's (1999). They were required to judge whether two targets were the same or different. Performance was equivalent whether the targets appeared within the same or within different grouped-UC regions. These results replicated Watson and Kramer's finding that no object-based effect is obtained in a divided-attention task when targets form separate single-UC regions.

Experiment 2 was a variant of Egly, Driver, and Rafal's (1994) spatial-cuing paradigm. The subjects were required to categorize a target appearing at a precued location on most of the trials. Cue-to-target SOA was 100, 200, or $300 \mathrm{msec}$. On invalid-cue trials, the target appeared either within the same or within a different grouped-UC region as the cue. Object-based effects were significant, and their magnitude did not vary as a function of SOA.

Experiment 3 was identical to Experiment 1, except that the targets appeared one at a time instead of simultaneously. Between-targets SOA was 100, 200, or $300 \mathrm{msec}$. Object-based effects were obtained at the short SOAs (100 and $200 \mathrm{msec}$ ), not at the long SOA.

In Experiment 4, the subjects had to categorize a target, which appeared at a $100 \%$ validly cued location, while ignoring a distractor that appeared on two thirds of the trials and was either compatible or incompatible with the target. The presence of the distractor slowed performance and produced compatibility effects. However, there were no object-based effects - that is, both the interference and the compatibility effects had the same magnitude whether the distractor appeared in the same or in a different grouped$\mathrm{UC}$ region as the target.

The most parsimonious account for the results of all four experiments is that a necessary condition for obtaining object-based effects with grouped-UC objects is the need for an attentional shift. Indeed, in Experiments 1 and 4 and in Experiment 3 (300-msec SOA condition), where no object-based effects were found, no shift of attention was required, since attention remained either distributed broadly or focused on a precued location. In Experiment 2 and in Experiment 3 (100- and 200-msec SOA conditions), where object-based effects were observed, the subjects had to shift their attention from the cue to the target on invalid-cue trials and from the first to the second target, respectively.

Other factors that can provide an alternative explanation for the difference in results in one pair of experiments can be dismissed on the basis of the results obtained in the other experiments. For instance, a spatial precue was used to direct attention in Experiment 2 (object-based effects), but not in Experiment 1 (no object-based effects ). How- 
ever, no object-based effects were obtained in Experiment 4 either, where a spatial precue was present. Attention was distributed in Experiment 1 (no object-based effect) and was focused in Experiments 2 and 3 (objectbased effects). However, attention was also focused in Experiment 4, where no object-based effects were found. The subjects had to judge the size of one target in Experiment 2 (object-based effects) or to compare the sizes of two targets in Experiment 1 (no object-based effects). However, no object-based effects were obtained in Experiment 4 , where the subjects had to categorize one target, and object-based effects were obtained in Experiment 2, where the subjects had to compare two targets. Finally, two single-UC regions were simultaneously present in the target displays of Experiments 1 and 4 (no object-based effect), whereas only one was present in Experiment 2 (object-based effects). However, object-based effects were also observed in Experiment 3, where, although asynchronously onset, the two targets were present in the target display.

As we noted earlier, the literature is replete with reports of object-based effects, obtained with various paradigms. On the basis of the idea proposed here that the need for a shift of attention is the necessary condition for obtaining object-based effects with grouped-UC objects, what predictions can be derived for the divided-attention, response competition, and spatial-cuing paradigms reviewed earlier, and how do these predictions fare when tested against the existing literature?

Divided-attention tasks, in which subjects make judgments concerning two targets that either belong or do not belong to the same perceptual group, should yield objectbased effects, because subjects typically need to shift their attention between the two targets in order to be able to compare them. This has been confirmed in several studies (e.g., Baylis \& Driver, 1993; Lavie \& Driver, 1996, Experiments 1-3). To our knowledge, Watson and Kramer's (1999) study and the present Experiment 1 are the only reports of no object-based effects with a divided-attention task. We suggested that in some divided-attention tasks, subjects may use alternative strategies-for example, subitizing or symmetry judgments - which may allow them to perform the task while keeping their attention widely distributed, with no need for shifts between targets and, thus, no object-based effects. Although this interpretation of the reported failures to obtain object-based effects is in line with the literature on subitizing (e.g., Trick \& Pylyshyn, 1993) and symmetry judgments (e.g., Baylis \& Driver, 1994), it would be useful to test it directly in further research.

With Eriksen's (Eriksen \& Hoffman, 1973) flanker task, in which shifts of attention are typically not required, objectbased effects with grouped-UC objects have been reported in some studies (Baylis \& Driver, 1992; Driver \& Baylis, 1989; Kramer \& Jacobson, 1991), but not in others (Berry \& Klein, 1993; Kramer, Tham, \& Yeh, 1991; Shomstein \& Yantis, 2002). The former are, thus, inconsistent with the claim made in this paper (note that they are also inconsis- tent with Watson and Kramer's, 1999, idea that no objectbased effects are to be found with grouped-UC objects). Baylis and Driver's (1992) findings, as well as those of Driver and Baylis (1989), have not been replicated (see Berry \& Klein, 1993; Kramer et al., 1991). Kramer and Jacobson presented their subjects with one central line (the target) flanked by two lines (the distractors). Subjects had to respond whether the target line was dotted or dashed. The distractors were either compatible or incompatible with the target. Kramer and Jacobson found larger compatibility effects with distractors grouped with the target by connectedness, which we would expect, since the target and the distractors belonged to the same UC region (Experiments 1 and 3), but also when they formed distinct single-UC regions and were grouped by common color (Experiment 2). Kramer and Jacobson suggested that "changes in the response-compatibility effect as a function of color coding could be interpreted in terms of either changes in visual confusability across conditions or perceptual grouping" (p. 276). They also recognized that the confusability hypothesis could not be decisively ruled out on the basis of their data (p. 277). There are several differences between Kramer and Jacobson's stimuli (Experiment 2) and the ones used in the present paper. For instance, the space between the target and the distractors was empty, whereas here, this space was perceived as being an object part distinct from the background. They used very small separations $\left(0.25^{\circ}\right.$ and $\left.1^{\circ}\right)$, whereas our targets and distractors were distant by more than $8^{\circ}$. The systematic investigation of which factors may explain the difference between the results of the present Experiment 4 and Kramer and Jacobson's results could provide valuable new insights into the conditions in which object-based effects are obtained.

Consistent with the present proposal, however, Shomstein and Yantis (2002) reported no object-based effect, using a variant of Eriksen's (Eriksen \& Hoffman, 1973) flanker task (Experiments 1-4). Subjects judged the shape of a central letter flanked by either two compatible or two incompatible distractors. The magnitude of the compatibility effect was not affected by whether or not the target and the distractors were in the same or in different objects. In a control experiment with the same objects (Experiment 5), the target and three distractors appeared after a cue was flashed in the center of the display. The target always appeared in one of four intermediate locations relative to the center, whereas the distractors appeared in either close or far locations. Thus, attention had to be shifted from the cue to the target location. Compatibility effects were smaller when the target appeared in the same object as the cue, rather than in a different object. Shomstein and Yantis concluded that when alternative locations must be scanned in search for the target, owing to spatial uncertainty, attention first inspects locations within the same object. However, in contradiction with our hypothesis and with the same-object prioritization idea, they found no main effect of object. It may be significant that the control experiment differed from the preceding experiment in numerous 
aspects. For instance, the target was defined by its position relative to the center, but also relative to the object's end, which may have made object structure task relevant; the same- versus different-object relation pertained to the cue and the target, whereas in preceding experiments it pertained to the distractors and the target. The inclusion of a cue and the higher number of distractors also introduced differences in the stimuli used. Thus, it is difficult to conclude whether the critical difference between the control and the other experiments was the need for attention to scan the displays, the need to shift attention, or any of the abovementioned differences.

With Egly, Driver, and Rafal's (1994) spatial-cuing paradigm, object-based effects are expected because attention has to be shifted between the cued location and the target location on invalid-cue trials. This prediction has been supported in the numerous studies that used variants of Egly, Driver, and Rafal's procedure (e.g., Abrams \& Law, 2000; Egly, Driver, \& Rafal, 1994; Lamy \& Tsal, 2000; Moore et al., 1998; Vecera, 1994). In this paper, we have also provided evidence showing that the object-based cost observed in these experiments is a cost of shifting attention between two different perceptual groups. Two alternative accounts for this effect can be found in the literature.

Some authors (e.g., Davis et al., 2000; Driver \& Baylis, 1998) have proposed that attention spreads from the cued location to the other locations grouped with it during the cue-to-target interval. The results of Experiment 2 argue against this possibility, since they show that the objectbased effect remains constant in magnitude whether the time available for attention to propagate is short $(100 \mathrm{msec})$ or long (300 msec).

Recently, it has been suggested that the object-based cost observed in Egly, Driver, and Rafal's (1994) paradigm results from a prioritization strategy in which same-object locations are scanned before different-object locations (e.g., Avrahami, 1999; Moore, Yantis, Vaughan, \& Handwerker, 2000; Shomstein \& Yantis, 2002). This hypothesis does not provide a natural account for the results reported here. In Experiment 3, in which the subjects had to compare two targets presented one after the other, objectbased effects were obtained with 100- and 200-msec SOAs, but not with 300-msec SOAs. The preferential scanning hypothesis does not predict that, in this situation, objectbased effects should interact with SOA in the way we observed. If there is a tendency to search potential target locations first within the same object, this tendency should be revealed at all SOAs.

The present interpretation of Egly, Driver, and Rafal's (1994) results is congruent with Egly, Driver, Rafal, and Starrveveld's (1994) work. They used Egly, Driver, and Rafal's spatial-cuing paradigm with split-brain patients and found object-based effects with invalidly cued targets only when these were presented in the right hemifield, but not in the left hemifield. They concluded that the left hemisphere controls shifts of attention between objects, which is consistent with our interpretation of the object-based effect as a cost in shifting attention between objects.
In principle, the absence of an object-based effect for left-hemifield targets can also be accommodated by the two alternative interpretations mentioned earlier, if one assumes that the left hemisphere is responsible for the mechanism by which attention spreads to regions grouped with the current focus of attention or for assigning a higher processing priority to locations within the same object. A common implication of these possibilities is that the left hemisphere is responsible for a performance advantage on same-object trials (owing to spreading or prioritization), rather than for a performance cost on different-object trials (owing to a need to shift attention between objects). Accordingly, they predict that this advantage should be wiped out for left-hemifield targets. In fact, Egly, Driver, Rafal, and Starrveveld found the RT costs for invalidcue trials, relative to valid-cue trials, to be small on sameand different-object trials for left-hemifield targets and equally small on same-object trials, but larger on different-object trials, for right-hemifield targets. This pattern of results is consistent with the interpretation of the object-based effect as a cost in shifting attention between objects, but not as a same-object advantage owing to spreading or prioritization.

In the present study, we explored the relevance of attentional shifts to the object-based selection issue only with grouped-UC objects. The findings presented by Watson and Kramer (1999) suggest that one cannot select part of a single-UC region without selecting the whole single$\mathrm{UC}$ region. On the basis of this idea, one may expect objectbased effects to be observed whether or not the task requires shifts of attention between single-UC objects. Although further research should establish this point, this would imply that the mechanisms revealed in object-based effects are very different for single-UC objects and for grouped-UC objects.

On the one hand, finding object-based effects with single-UC objects would result from the fact that single$\mathrm{UC}$ regions are the smallest parts that can be selectively attended. That is, single-UC regions are selected as indivisible chunks, and selecting one part of a single-UC region therefore entails the mandatory selection of its other parts. On the other hand, finding object-based effects with grouped-UC objects would result from the fact that shifting attention between single-UC regions belonging to different perceptual groups (or grouped-UC regions) incurs a cost.

In this paper, we have established the existence of this cost but have not explored its nature. Humphreys's (1998) dual-coding account of neural representation of objects in space suggests an interesting possibility. Drawing from his work with neurologically impaired patients, he proposed that at least two forms of object representation exist in the brain, which are realized in parallel by the visual system: one in which elements are coded as parts of a single object within the ventral visual stream (within-object representation) and one in which elements are coded independently within the dorsal visual stream (betweenobjects representation). The latter claim has been sup- 
ported by Egly, Driver, and Rafal's (1994) finding that patients with parietal lesions show an abnormal pattern of performance when required to shift their attention between objects.

If we apply Palmer and Rock's (1994) framework to Humphreys's (1998) scheme, it follows that a groupedUC object is coded in parallel as one single object in the within-object code and as several different objects (one for each single-UC region) in the between-objects code. When attention is shifted between two different single-UC regions within the same grouped-UC object, the coordinates of the attentional locus need to be updated in the between-objects code, but not in the within-object code. In contrast, when attention is shifted between single-UC regions belonging to separate grouped-UC objects, both codes need to be updated. The performance cost of shifting attention between grouped-UC regions, reported in the present experiments, may thus derive from the additional updating of the within-object code. No such updating procedures are necessary when attention remains widely distributed throughout the task.

\section{REFERENCES}

Abrams, R. A., \& Law, M. B. (2000). Object-based visual attention with endogenous orienting. Perception \& Psychophysics, 62, 818-833.

Atchley, P., \& Kramer, A. F. (1998, November). Spatial and objectbased attentional selection in three-dimensional space. Paper presented at the 39th Annual Meeting of the Psychonomic Society, Dallas.

Avrahami, J. (1999). Objects of attention, objects of perception. Perception \& Psychophysics, 61, 1604-1612.

BAYlis, G. C., \& Driver, J. (1992). Visual parsing and response competition: The effect of grouping factors. Perception \& Psychophysics, 51, 145-162.

Baylis, G. C., \& Driver, J. (1993). Visual attention and objects: Evidence for hierarchical coding of location. Journal of Experimental Psychology: Human Perception \& Performance, 19, 451-470.

BAYlis, G. C., \& Driver, J. (1994). Parallel computation of symmetry but not repetition within single visual shapes. Visual Cognition, $\mathbf{4}$, 377-400.

Behrmann, M., Zemel, R, \& Mozer, M. (1998). Object-based attention and occlusion: Evidence from normal subjects and a computational model. Journal of Experimental Psychology: Human Perception \& Performance, 24, 1011-1036.

BERRY, G., \& KLEIN, R. (1993). Does motion-induced grouping modulate the flanker compatibility effect? A failure to replicate Driver \& Baylis. Canadian Journal of Experimental Psychology, 47, 714-729.

Broadbent, D. E. (1982). Task combination and selective intake of information. Acta Psychologica, 50, 253-290.

Bundesen, C. (1990). A theory of visual attention. Psychological Review, 97, 523-547.

Chen, Z (1998). Switching attention within and between objects: The role of subjective organization. Canadian Journal of Experimental Psychology, 52, 7-16.

Davis, G., Driver, J., Pavani, F., \& Shepherd, A. (2000). Reappraising the apparent costs of attending to two separate visual objects. Vision Research, 40, 1323-1332.

Downing, C. J., \& Pinker, S. (1985). The spatial structure of visual attention. In M. I. Posner \& O. S. M. Marin (Eds.), Attention and performance XI (pp. 171-187). Hillsdale, NJ: Erlbaum.

DrIVER, J., \& BAY LIS, G. C. (1989). Movement and visual attention: The spotlight metaphor breaks down. Journal of Experimental Psychology: Human Perception \& Performance, 15, 448-456.

Driver, J., \& BAYLIS, G. C. (1998). Attention and visual object segmentation. In R. Parasuraman (Ed.), The attentive brain (pp. 299-325). Cambridge, MA: MIT Press.
Duncan, J. (1984). Selective attention and the organization of visual information. Journal of Experimental Psychology: General, 113, 501517.

Duncan, J., \& Humphreys, G. W. (1989). Visual search and stimulus similarity. Psychological Review, 96, 433-458.

Duncan, J., \& Humphreys, G. W. (1992). Beyond the search surface: Visual search and attentional engagement. Journal of Experimental Psychology: Human Perception \& Performance, 18, 578-588.

EgETh, H. E., \& YANTIS, S. (1997). Visual attention: Control, representation and time course. Annual Review of Psychology, 48, 269-297.

Egly, R, Driver, J., \& RAFAL, R. D. (1994). Shifting visual attention between objects and locations: Evidence from normal and parietal lesion subjects. Journal of Experimental Psychology: General, 123, 161-177.

Egly, R., Driver, J., Rafal, R. D., \& Starrveveld, Y. (1994). Covert orienting in the split brain reveals hemispheric specialization for object-based attention. Psychological Science, 5, 380-383.

ERIKSEn, C. W., \& Hoffman, J. E. (1973). The extent of processing of noise elements during selective encoding from visual displays. Perception \& Psychophysics, 14, 155-160.

ERIKSEN, C. W., \& ST. JAMES, J. D. (1986). Visual attention within and around the field of focal attention. Perception \& Psychophysics, 40 , 225-240.

ERIKSEN, C. W., \& YeH, Y. Y. (1985). Allocation of attention in the visual field. Journal of Experimental Psychology: Human Perception \& Performance, 11, 583-597.

Humphreys, G. W. (1998). Neural representations of objects in space: A dual coding account. In G. W. Humphreys, J. Duncan, \& A. Treisman (Eds.), Attention, space and action: Studies in cognitive neuroscience (pp. 165-182). New York: Oxford University Press.

Kahneman, D., \& Treisman, A. (1984). Changing views on automaticity. In R. Parasuraman \& D. R. Davies (Eds.), Varieties of attention (pp. 29-62). Orlando, FL: Academic Press.

KANWISHer, N., \& Driver, J. (1992). Objects, attributes, and visual attention: Which, what, and where. Current Directions in Psychological Science, 1, 26-31.

Kramer, A. F., \& Jacobson, A. (1991). Perceptual organization and focused attention: The role of objects and proximity in visual processing. Perception \& Psychophysics, 50, 267-284.

Kramer, A. F., Tham, M.-P., \& Yeh, Y.-Y. (1991). Movement and focused attention: A failure to replicate. Perception \& Psychophysics, 50, 537-546.

Kramer, A. F., Weber, T. A., \& Watson, S. E. (1997). Object-based attentional selection: Grouped arrays or spatially invariant representations? Journal of Experimental Psychology: General, 126, 3-13.

LABERge, D., \& Brown, V. (1989). Theory of attentional operations in shape identification. Psychological Review, 96, 101-124.

LAMY, D. (2000). Object-based selection under focused attention: A failure to replicate. Perception \& Psychophysics, 62, 1272-1279.

LAMY, D., \& TSAL, Y. (2000). Object features, object locations and objectfiles: Which does selective attention activate and when? Journal of Experimental Psychology: Human Perception \& Performance, 26, 1387-1400.

Lamy, D., \& Tsal, Y. (2001). On the status of location in visual attention. European Journal of Cognitive Psychology, 13, 305-342.

Lavie, N., \& Driver, J. (1996). On the spatial extent of attention in object-based visual selection. Perception \& Psychophysics, 58, 12381251.

Moore, C. M., Yantis S., \& Vaughan, B. (1998). Object-based visual selection: Evidence from perceptual completion. Psychological Science, 9, 104-110.

Moore, C. M., Yantis, S., Vaughan, B., \& Handwerker, D. A. (2000). Functional differences between space-based and object-based visual selection. Manuscript submitted for publication.

Neisser, U. (1967). Cognitive psychology. New York: AppletonCentury-Crofts.

PAlmer, S., \& Rock, I. (1994). Rethinking perceptual organization: The role of uniform connectedness. Psychonomic Bulletin \& Review, 1, 29-55.

Posner, M. I., SNyder, C. R., \& Davidson, B. J. (1980). Attention and the detection of signals. Journal of Experimental Psychology: General, 109, 160-174. 
SAIKI, J. (2000). Occlusion, symmetry, and object-based attention: Comment on Behrmann, Zemel, and Mozer (1998). Journal of Experimental Psychology: Human Perception \& Performance, 26, 424433.

Shomstein, S., \& Yantis, S. (2002). Object-based attention: Sensory modulation or priority setting? Perception \& Psychophysics, 64, 4151.

Treisman, A., Kahneman, D., \& Burkell, J. (1983). Perceptual objects and the cost of filtering. Perception \& Psychophysics, 33, 527532.

Trick, L. M., \& Pylyshyn, Z W. (1993). What enumeration studies can show us about spatial attention: Evidence for limited capacity preattentive processing. Journal of Experimental Psychology: Human Perception \& Performance, 19, 331-351.

VECERA, S. P. (1994). Grouped locations and object-based attention: Comment on Egly, Driver, \& Rafal (1994). Journal of Experimental Psychology: General, 123, 316-320.

VECERA, S. P. (1997). Grouped arrays versus object-based representations: Reply to Kramer et al. (1997). Journal of Experimental Psychology: General, 126, 14-18.

Vecera, S. P., \& Farah, M. J. (1994). Does visual attention select objects or locations? Journal of Experimental Psychology: General, 123, 146-160.

Watson, S. E., \& Kramer, A. F. (1999). Object-based visual selective attention and perceptual organization. Perception \& Psychophysics, 61, 31-49.

Zemel, R. S., Behrmann, M., Mozer, M. C., \& Bavelier, D. (2000). Experience-dependent perceptual grouping and object-based attention. Manuscript submitted for publication.

\section{NOTES}

1. Parsed-UC regions, which are smaller representations segregated by parsing single-UC regions at points of concave discontinuities, were also included in their framework. These will not be discussed in the present paper, but the relationship between single-UC and grouped-UC regions and the relationship between parsed-UC and single-UC regions are theoretically equivalent.

2. Null effects are typically not reported, and only a few authors have attempted to explore the boundary conditions of object-based selection. As a consequence, in order to probe whether or not the proposed framework can accommodate the existing body of research on object-based selection, we can survey mainly whether those tasks in which objectbased effects with grouped-UC objects were obtained involved shifts of attention. The available literature against which we can test the other part of our claim-namely, whether tasks in which no shifts of attention were required indeed yielded no object-based effects-is rather scarce and is limited to flanker tasks.

(Manuscript received August 21, 2000;

revision accepted for publication February 27, 2001.) 